\title{
On the Characteristics of Linear-Phase Roll Vortices under a Moving Hurricane Boundary Layer
}

\author{
KUN GAO ${ }^{\mathrm{a}}$ AND ISAAC GINIS \\ Graduate School of Oceanography, University of Rhode Island, Narragansett, Rhode Island
}

(Manuscript received 27 November 2017, in final form 12 April 2018)

\begin{abstract}
Previous theoretical and numerical studies only focused on the formation of roll vortices (rolls) under a stationary and axisymmetric hurricane. The effect of the asymmetric wind structure induced by the storm movement on the roll characteristics remains unknown. In this study, we present the first attempt to investigate the characteristics of linear-phase rolls under a moving hurricane by embedding a linear twodimensional (2D) roll-resolving model into a 3D hurricane boundary layer model. It is found that the roll horizontal wavelength under the moving hurricane is largely determined by the radial-shear-layer depth, defined as the thickness of the layer with positive radial wind shear. The horizontal distribution of the roll wavelength resembles the asymmetric pattern of the radial-shear-layer depth. Interestingly, the roll growth rate is not only affected by the radial wind shear magnitude alluded to in previous studies but also by the radial-shear-layer depth. A deeper (shallower) radial shear layer tends to decrease (increase) the roll growth rate. Such an effect is due to the presence of the bottom boundary. The bottom boundary constrains the lowerlevel roll streamlines and reduces the efficiency of rolls in extracting kinetic energy from the radial shear. This effect is more pronounced under a deeper shear layer, which favors the formation of larger-size rolls. This study improves the understanding of the main factors affecting the structure and growth of rolls and will provide guidance for interpreting the spatial distribution of rolls under realistic hurricanes in observations and high-resolution simulations.
\end{abstract}

\section{Introduction}

Numerous observational studies (Wurman and Winslow 1998; Morrison et al. 2005; Lorsolo et al. 2008; Zhang et al. 2008; Ellis and Businger 2010) indicate that roll vortices (rolls), which are coherent kilometer-scale eddies roughly aligned in the hurricane tangential wind direction, frequently occur in the hurricane boundary layer (HBL). These eddies significantly contribute to the vertical transports of momentum and entropy in the HBL (e.g., Zhang et al. 2008) and may have an important impact on the hurricane structure and intensity (Gao and Ginis 2016; Gao et al. 2017). Studies focusing on the formation mechanisms of rolls [e.g., analytical study: Foster (2005); two-dimensional model: Gao and Ginis (2014, hereafter GG14, 2016); large-eddy simulations: Nakanishi and Niino (2012) and Wang and Jiang (2017)] are generally

\footnotetext{
${ }^{a}$ Current affiliation: Program in Atmospheric and Oceanic Sciences, Princeton University, Princeton, New Jersey.
}

Corresponding author: Kun Gao, kun.gao@noaa.gov in agreement that the rolls in the HBL are generated by the inflection-point instability associated with the vertical distribution of the hurricane radial wind. Particularly, GG14 suggested that the roll growth rate depends on the magnitude of radial wind shear and the roll horizontal wavelength depends on the height of the shear layer. All these previous studies focused on the rolls under a stationary and axisymmetric hurricane. The effect of the hurricane wind asymmetry induced by the storm movement on the roll formation remains unknown. In this study, we explore for the first time the characteristics of linear-phase rolls under a moving hurricane in a numerical model. The term "linear phase" refers to the period when the roll velocities grow exponentially with time but remain at least one order smaller than the mean winds, and thus, the nonlinear terms in their governing equations are negligible. Similar to GG14, this study focuses on understanding how the mean flow affects the roll characteristics, which constitutes a necessary step toward understanding how the rolls affect the mean flow once they enter the nonlinear phase (Gao and Ginis 2016). Our main objective in 
this study is to understand how the asymmetric radial wind structure in the HBL affects the spatial scale and growth rate of rolls.

\section{Method}

The methodology in this study is similar to GG14. To resolve the linear-phase rolls, we embed a linear version of the two-dimensional (2D) single-grid roll-resolving model (SRM) into a diagnostic HBL model. The linearroll equations are the same as in GG14 [see their (7)-(10)] and will not be shown here. To account for the effect of storm movement, we replace the axisymmetric HBL model in GG14 with a three-dimensional (3D) HBL model. Similar to previous studies (Kepert and Wang 2001; GG14), the diagnostic HBL model is formulated under the assumption that the HBL wind fields are in a steady state under an imposed upper vortex. The diagnosed secondary flow (radial and vertical winds) is entirely caused by the surface friction. The equations for the mean HBL wind components in the Cartesian coordinates are given as follows:

$$
\begin{aligned}
& \frac{\partial U}{\partial t}+U \frac{\partial U}{\partial x}+V \frac{\partial U}{\partial y}+W \frac{\partial U}{\partial z}-f V=-\frac{1}{\rho_{0}} \frac{\partial P}{\partial x} \\
&+\frac{\partial}{\partial z}\left(K_{z} \frac{\partial U}{\partial z}\right) \\
& \frac{\partial V}{\partial t}+U \frac{\partial V}{\partial x}+V \frac{\partial V}{\partial y}+W \frac{\partial V}{\partial z}+f U=-\frac{1}{\rho_{0}} \frac{\partial P}{\partial y} \\
&+\frac{\partial}{\partial z}\left(K_{z} \frac{\partial V}{\partial z}\right) \\
& \frac{\partial U}{\partial x}+\frac{\partial V}{\partial y}+\frac{\partial W}{\partial z}=0
\end{aligned}
$$

where $U, V$, and $W$ are the wind components in the $x, y$, and $z$ directions, respectively; $f$ is the Coriolis parameter at $20^{\circ} \mathrm{N} ; P$ is the pressure; and $K_{z}$ is the vertical diffusion parameterized as in GG14 [see their (5) and (6)]. The asymptotic mixing length is set to $40 \mathrm{~m}$ in this study. ${ }^{1}$ The surface-layer parameterization in the $3 \mathrm{D}$ HBL model is the same as in GG14. The three wind components are defined on nonstaggered grids. The upwind finitedifference scheme is used to discretize the advection

\footnotetext{
${ }^{1}$ The impact of asymptotic mixing length on the mean wind and roll characteristics under the axisymmetric HBL has been discussed in GG14. The main findings hold under the moving HBL. The choice of asymptotic mixing length does not have any qualitative impact on the main conclusions presented in this study.
}

terms in (1) and (2). The $W$ wind component is diagnosed based on integrating the horizontal divergence upward from the surface.

Similar to Kepert and Wang (2001), we adopt a translating coordinate system: the mesh moves at the same speed as the hurricane so that the hurricane is stationary relative to the mesh (the storm center is the origin of the $x$ and $y$ coordinates). The wind at the top boundary (set to the $3-\mathrm{km}$ height) and the pressure gradients in (1) and (2) throughout the entire column are prescribed. The wind field at the top boundary consists of two components: a spatially uniform environmental flow (i.e., the storm translation speed), denoted by $U_{g}$ (subscript $g$ stands for geostrophic), and the axisymmetric hurricane tangential wind field. The pressure gradient terms, which are held fixed in time and vertically uniform, are obtained by solving the balanced equations in (1) and (2) without considering the vertical advection and turbulent diffusion terms.

Two experiments are conducted. The storm moves westward at a constant speed of $5 \mathrm{~m} \mathrm{~s}^{-1}\left(U_{g}=-5 \mathrm{~m} \mathrm{~s}^{-1}\right)$ in experiment MOVE and remains stationary in experiment STAT $\left(U_{g}=0 \mathrm{~m} \mathrm{~s}^{-1}\right)$. All other parameters are identical. The HBL model domain is set to $1000 \mathrm{~km}$ wide in both the $x$ and $y$ directions with a $10-\mathrm{km}$ horizontal and a $30-\mathrm{m}$ vertical grid spacing. In both experiments, the Holland (1980) parametric wind model is used to specify the hurricane tangential wind at the top boundary. As in GG14, the hurricane wind reaches a maximum value of $39 \mathrm{~m} \mathrm{~s}^{-1}$ at a radius of $40 \mathrm{~km}$, and the parameter $B$ is set to 1.3 .

In each experiment, the $3 \mathrm{D} \mathrm{HBL}$ model is initialized with vertically uniform wind and run for $24 \mathrm{~h}$ to obtain a well-adjusted steady-state wind field. We then embed the linear SRM into the 3D HBL model at every horizontal grid point within an annular area between 40- and $100-\mathrm{km}$ radii. The SRM is not embedded within radius of maximum wind (RMW) because the assumption that the mean-flow profiles are horizontally uniform within the SRM domain (GG14) may not be valid at small radii. The configuration of the linear SRM is identical to GG14 (see their sections 2c and 2d). For simplicity, the 2D SRM mesh is positioned perpendicular to the storm-relative tangential wind at each selected grid point. Also for simplicity, we assume the HBL is neutrally stratified and thus exclude the effect of stratification on rolls in this study.

\section{Results}

\section{a. Characteristics of linear-phase rolls}

Figure 1 shows the steady-state storm-relative (total minus $U_{g}$ ) wind in the HBL from experiment MOVE. 

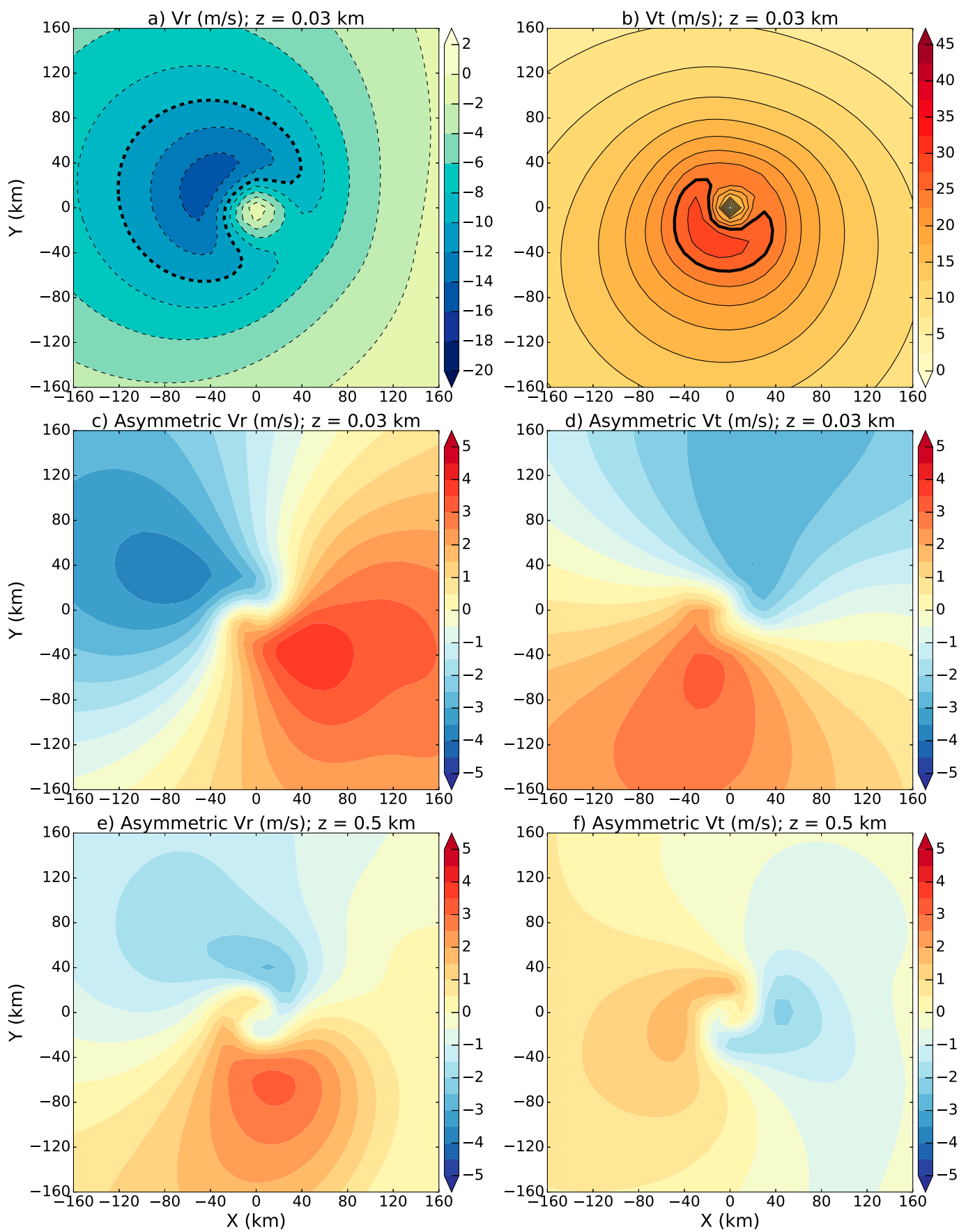

FIG. 1. Steady-state wind distribution in experiment MOVE obtained by the diagnostic HBL model. (top) Stormrelative (a) radial and (b) tangential winds at 30-m height; the contour interval is $2 \mathrm{~m} \mathrm{~s}^{-1}$ for the radial wind (thick dashed line: $-10 \mathrm{~m} \mathrm{~s}^{-1}$ contour ) and $2.5 \mathrm{~m} \mathrm{~s}^{-1}$ for the tangential wind (thick solid line: $30 \mathrm{~m} \mathrm{~s}^{-1}$ contour ). (middle) Storm-relative asymmetric (c) radial and (d) tangential winds at 30-m height. (bottom) (e),(f) As in (c) and (d), respectively, but for $0.5-\mathrm{km}$ height.

The storm movement causes stronger (weaker) surface friction on the right (left) side of the storm, which leads to an asymmetry in the wind field. The near-surface radial and tangential wind distributions (Figs. 1a,b) are consistent with those in previous studies using linear (Kepert 2001; see his Fig. 3) and nonlinear (Kepert and Wang 2001; see their Fig. 10) HBL models. The asymmetric wind components (Figs. 1c-f) are calculated by 

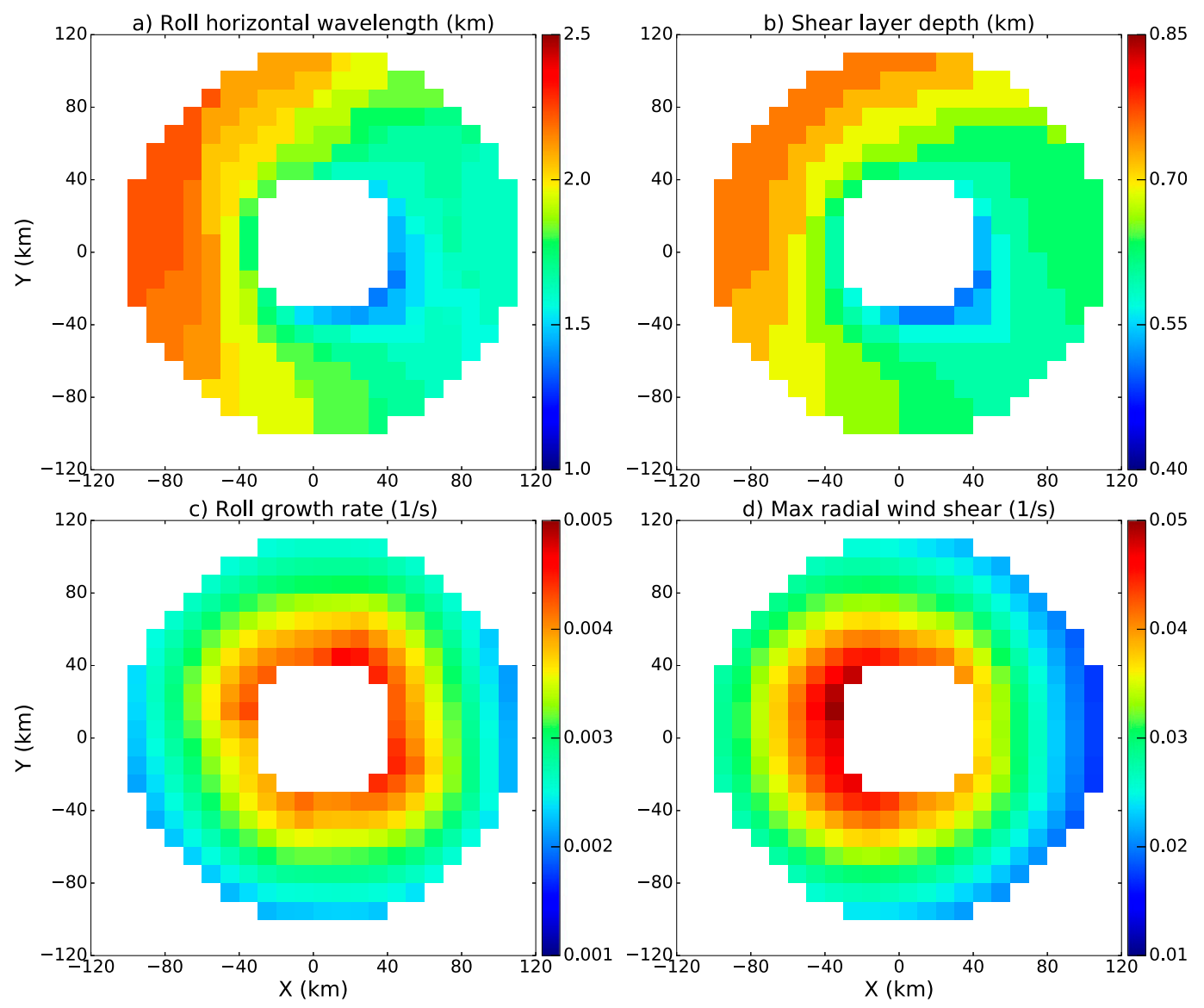

FIG. 2. Horizontal distributions of (a) roll horizontal wavelength, (b) shear-layer depth, (c) roll growth rate, and (d) maximum radial wind shear in experiment MOVE.

subtracting the azimuthal-averaged components from the total. The asymmetric components are dominated by the wavenumber- 1 structure, which rotates anticyclonically and decays in magnitude with height, consistent with the characteristics of the frictionally stalled inertia wave identified by Kepert (2001).

We next examine the characteristics of rolls from experiment MOVE with a focus on their horizontal wavelength (an indicator of their spatial scale) and growth rate (an indicator of their efficiency in extracting the kinetic energy from the mean shear). ${ }^{2}$ GG14 indicated that the rolls gain kinetic energy (KE) from the positive radial wind shear during their formation. In this study, we define the shear-layer depth as the thickness of the near-surface layer with positive radial wind shear

\footnotetext{
${ }^{2}$ The linear rolls resolved by the SRM can be represented in the normal-mode form. For example, the roll vertical velocity $w^{\prime}$ can be written as $w^{\prime}=\hat{w}(z) \exp i(k x-\omega t)$, where $k$ is the roll horizontal wavenumber; $\omega$ is a complex number and $\omega=\omega_{r}+i \omega_{i}$, where $\omega_{r}$ is the roll angular frequency and $\omega_{i}$ is the roll growth rate.
}

and use the maximum radial wind shear as a measure of the shear magnitude (see Fig. $4 \mathrm{~b}$ for representative radial wind shear profiles). ${ }^{3}$ Storm movement causes significant asymmetry in the horizontal distribution of roll wavelength, with larger wavelengths in the front of the storm (Fig. 2a) where the shear-layer depths are generally larger (Fig. 2b). Although the asymmetric pattern of roll wavelength does not match perfectly with the shear-layer depth, the result here suggests that the spatial scale of rolls is largely determined by the shear-layer depth, consistent with the finding of GG14 in the axisymmetric HBL. However, the roll growth rate (Fig. 2c) is not as well correlated with the magnitude of radial wind shear (Fig. 2d) as seen in the axisymmetric HBL experiment (GG14). The magnitude of radial wind shear has an apparent front-rear asymmetric pattern; however,

\footnotetext{
${ }^{3}$ The theoretical depth scale used in GG14 cannot adequately represent the asymmetric distribution of the shear-layer thickness under moving HBL. Therefore, we use the shear-layer depth as a depth-scale parameter.
} 
the roll growth rate does not. This indicates that besides the magnitude of the radial wind shear, there are possibly other factors affecting the roll growth rate.

To investigate this further, we compare the roll growth rates in experiments STAT and MOVE as a function of the maximum radial wind shear (Fig. 3). In experiment STAT, the roll growth rate increases approximately linearly with the increasing shear. However, the roll growth rate in MOVE, while increasing overall with the radial wind shear magnitude, has a large scatter around the best-fit line. Interestingly, if we separate the roll growth rates in MOVE into two groups based on the shear-layer depth at their formation location, most of the points where the shear-layer depth is larger (smaller) than that in STAT fall onto the lower (upper) side of the best-fit line. This suggests that the shear-layer depth has a strong influence on the roll growth rate. A shallower shear layer favors a larger roll growth rate.

We next examine three representative cases of roll formation, all occurring at the radius of maximum wind (Fig. 4). Case A is from experiment STAT, which serves as a reference case; cases $\mathrm{B}$ and $\mathrm{C}$ are selected from experiment MOVE at the grid points in front of and behind the storm, respectively. The radial wind profile in case $\mathrm{B}$ (case $\mathrm{C}$ ) is characterized by larger (smaller) shear and a deeper (shallower) shear layer than in case A (Figs. 4a,b). Among the three cases, the rolls in case B (case $\mathrm{C}$ ) have the largest (smallest) wavelength because of the largest (smallest) shear-layer depth (Figs. 4d-f). However, the rolls in case B (case C) have the smallest (largest) growth rate even though the radial wind shear is the strongest (weakest) (Fig. 4b).

To understand the difference in the roll growth rate in these cases, we next examine the shear production term in the roll KE budget equation [(11) in GG14], which is the dominant contributor to the growth of linear-phase rolls and written as

$$
-\overline{w^{\prime} u^{\prime}} \frac{\partial \bar{u}}{\partial z}
$$

where the overbar indicates the horizontal averaging within the SRM domain, $\overline{w^{\prime} u^{\prime}}$ is the horizontally averaged cross-roll momentum flux ( $w^{\prime}$ and $u^{\prime}$ constitute the roll overturning circulation), and $\bar{u}$ is the mean crossroll wind (i.e., the mean radial wind). Figure $4 \mathrm{c}$ shows the shear production terms when the rolls in the three cases have the same magnitude (measured by the domainaveraged roll $\mathrm{KE}$ ), respectively. The shear production in case B (case C) is the smallest (largest). A comparison of the $w^{\prime} u^{\prime}$ distribution within the shear layer (indicated by the dashed lines in Figs. 4d-f) suggests that the positive values of $w^{\prime} u^{\prime}$ are least (most) significant in case C

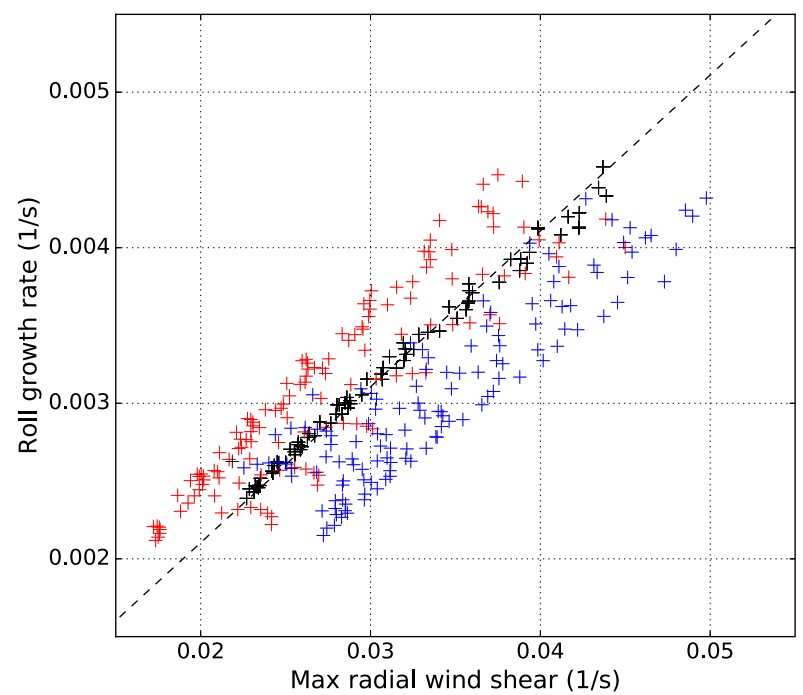

FIG. 3. The roll growth rate as a function of maximum radial wind shear in experiments STAT (shown as black) and MOVE (shown as red and blue). The dashed straight line shows the best-fit linear function of the results from experiment STAT. At a given horizontal grid point in the 3D HBL model, if the shear-layer depth in MOVE is smaller (larger) than that in STAT, the roll growth rate is marked as red (blue).

(case B), resulting in the largest (smallest) magnitude of $-\overline{w^{\prime} u^{\prime}}$ and thus the shear production. Therefore, we have identified that the $w^{\prime} u^{\prime}$ distribution in the shear layer is responsible for the differences in the roll growth rate. The sign and magnitude of $w^{\prime} u^{\prime}$ depend on the orientation of the lower-level roll streamlines ${ }^{4}$ (as indicated in Figs. 4d-f). The essential question remains: How does the shear-layer depth affect the lower-level roll streamline patterns?

\section{b. Elucidation of the roll structure and growth rate}

\section{1) EXPERIMENTS WITH REMOVED GEOMETRIC CONSTRAINT OF BOTTOM BOUNDARY}

In this section, we examine the geometric constraint on the near-surface roll streamlines imposed by the bottom boundary. Specifically, we postulate the following hypothesis to explain the relationship between the roll growth rate and the shear-layer depth: the rolls under a deeper shear layer have a larger vertical scale, and therefore, their lower-level streamlines are more significantly affected by the bottom boundary, resulting in the reduced growth rate. To validate this hypothesis,

\footnotetext{
${ }^{4}$ The cross-roll velocity $u^{\prime}$ and vertical velocity $w^{\prime}$ can be written as follows: $u^{\prime}=-\partial \psi^{\prime} / \partial z$ and $w^{\prime}=\partial \psi^{\prime} / \partial x$, where $\psi^{\prime}$ is the roll streamfunction.
} 

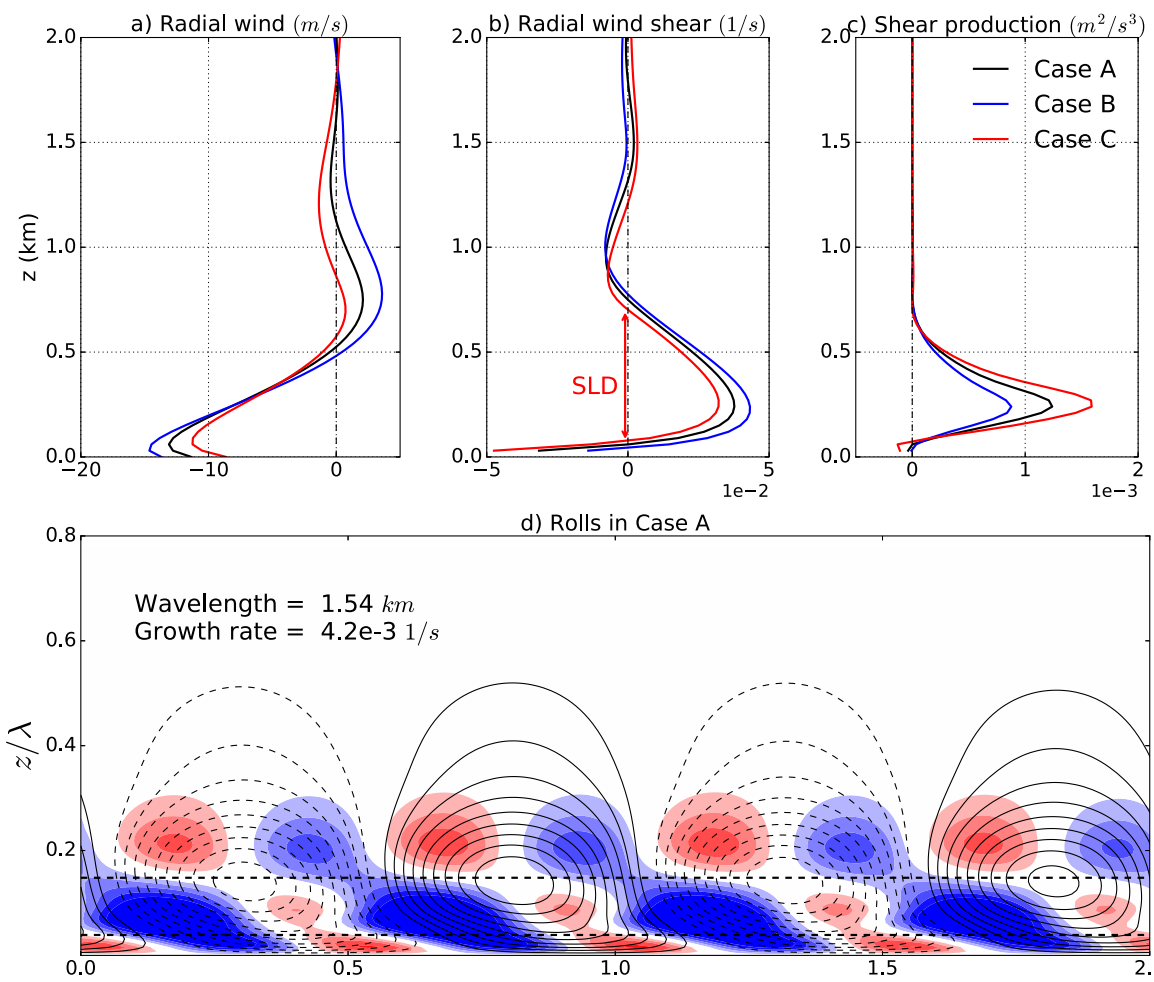

d) Rolls in Case A

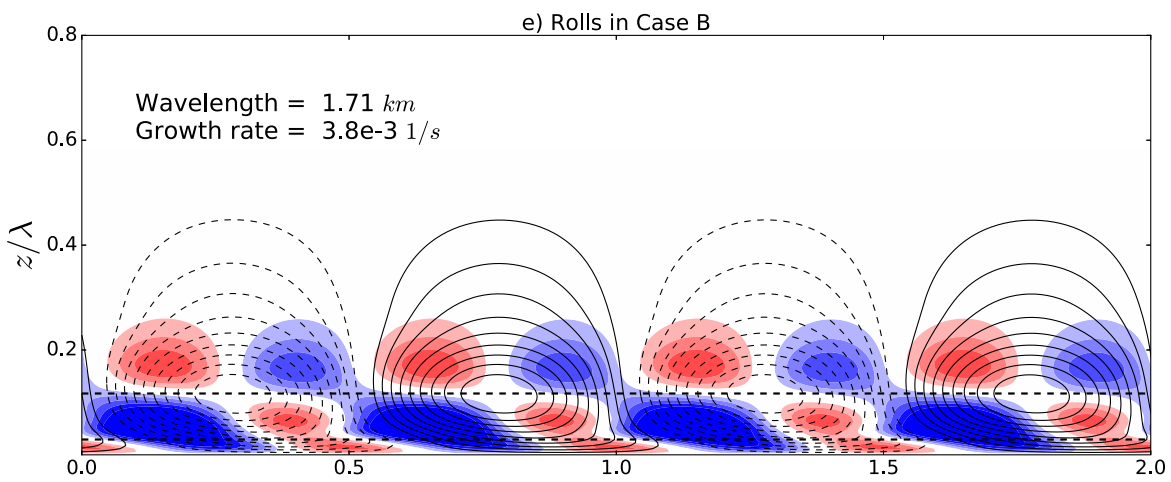

f) Rolls in Case C

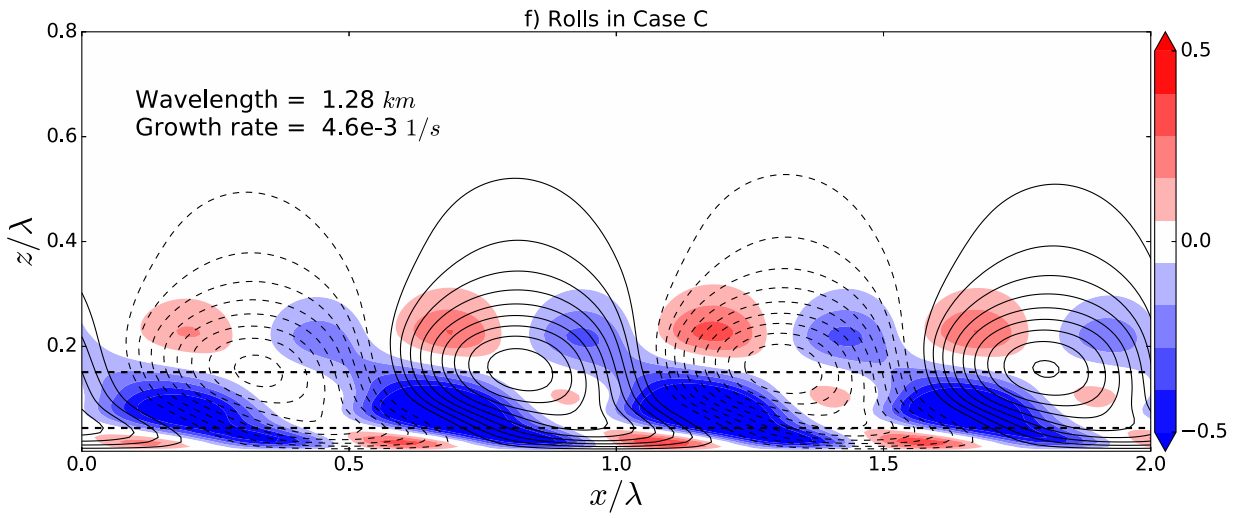

FIG. 4. (a) Vertical profiles of radial wind at RMW in experiments STAT (black) and MOVE (blue and red represent the grid points at the front and rear of the storm, respectively); (b) as in (a), but for radial wind shear; (c) as in (a), but for the shear production of roll overturning KE when the domain-averaged roll kinetic energy from the three cases are the same. (d)-(f) Structure of rolls from the three cases in the normalized coordinates ( $x$ and $z$ divided by the roll horizontal wavelength). The colored backgrounds represent normalized cross-roll momentum flux $w^{\prime} u^{\prime}$ (divided by its maximum absolute value); the contour lines represent the roll streamlines (solid contours show clockwise and dashed contours show counterclockwise circulations). The color bar ranges are from -0.5 to 0.5 to better show the positive values. The dashed lines in (d)-(f) indicate the range of the vertical layer with the most significant radial shear $(0.15-0.5 \mathrm{~km})$. 

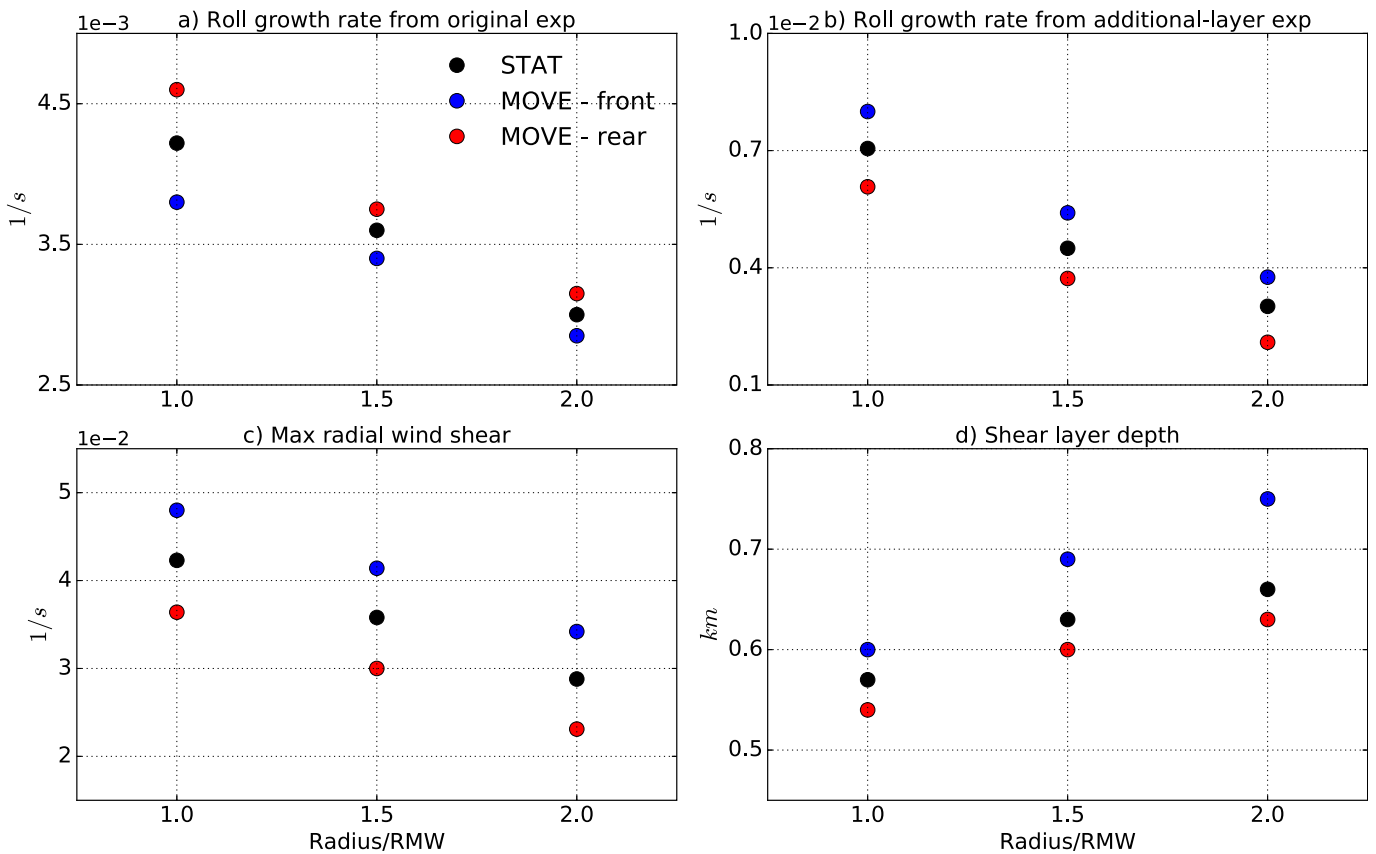

FIG. 5. (a) The roll growth rate at three selected radii (normalized by RMW): black shows the values from experiment STAT; blue and red show the values at the grid points at the front and rear of the storm from experiment MOVE, respectively. (b) As in (a), but for rolls from the additional-layer experiments. (c),(d) As in (a), but for the maximum radial wind shear and shear-layer depth, respectively.

we conducted additional experiments, in which we added a 3-km layer in the SRM below the original bottom boundary $(z=0 \mathrm{~km})$ to exclude the constraint of the bottom boundary on the roll streamlines. The mean cross-roll (radial) wind was set to zero in this additional layer, and thus, there was no additional energy source for the rolls in these new experiments ("additional layer" experiments). Figure 5 summarizes the results from the original and the additional-layer experiments ${ }^{5}$ at a few representative horizontal grid points. At a given radius, although driven by the strongest radial wind shear (Fig. 5c), the rolls in the front of the storm in experiment MOVE have the smallest growth rate $^{6}$ (Fig. 5a), which is likely due to the largest shear-layer depth (Fig. 5d). Figure 5b shows that after removing the constraint of the bottom boundary on the roll structure, the roll growth rate only depends on the magnitude of the radial wind shear. This confirms the hypothesis that the dependency of the roll growth rate on the shearlayer depth is due to the constraint of the bottom

\footnotetext{
${ }^{5}$ The rolls from the additional-layer experiments have structures similar to those in Fig. $6 \mathrm{c}$ and therefore are not shown.

${ }^{6}$ The difference in the $e$-folding growth time between rolls at the front and rear of the storm is relatively small, suggesting the storm movement does not significantly affect the occurring probability of rolls.
}

boundary on the roll structure. ${ }^{7}$ Also, the roll growth rate becomes larger in the additional-layer experiments than in the original experiments at all selected locations (Figs. 5a,b), suggesting that the constraint of the bottom boundary on the roll structure negatively affects the roll growth.

\section{2) EXPERIMENTS WITH HYPERBOLIC-TANGENT VELOCITY PROFILE}

To understand how the bottom boundary affects the roll streamlines and the roll growth rate, we introduce the effect of a boundary in the classic shear instability problem. In the following, we use the hyperbolic-tangent velocity profile that is often applied in shear instability studies (e.g., Carpenter et al. 2013) as the cross-roll mean flow in the linear SRM model. The velocity $u$ and vertical shear $s$ are given as follows (Fig. 6a):

$$
\begin{aligned}
& u(z)=\delta u \tanh \left(\frac{z}{h_{0}}\right), \\
& s(z)=s_{0}\left[1-\tanh ^{2}\left(\frac{z}{h_{0}}\right)\right],
\end{aligned}
$$

\footnotetext{
${ }^{7}$ The bottom boundary could also affect the roll growth rate via the surface friction. However, this is a very small term in the roll $\mathrm{KE}$ budget equation compared to the shear production (not shown).
} 

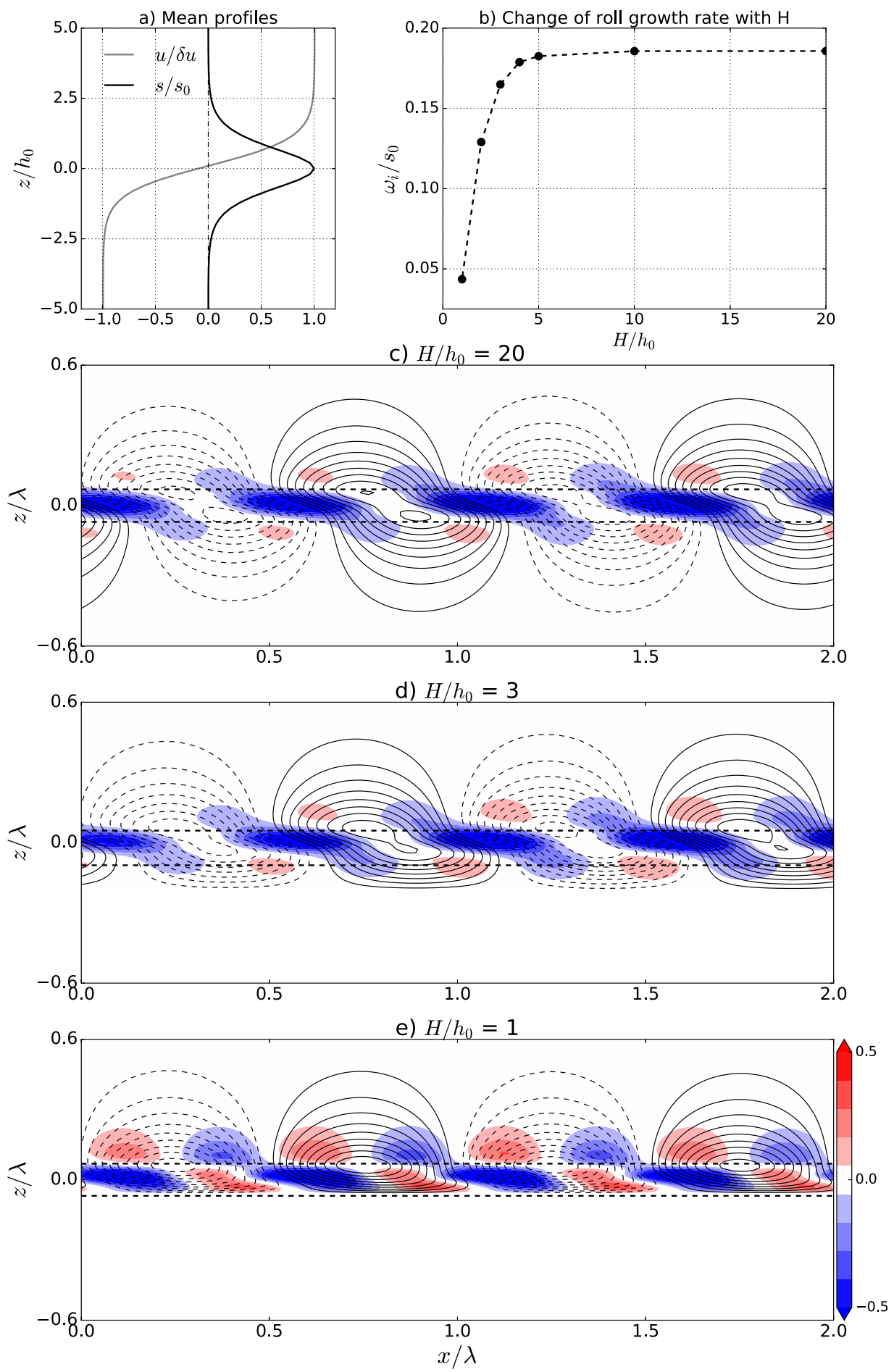

FIG. 6. (a) The velocity (normalized by $\delta u$; gray line) and shear (normalized by $s_{0}$; black line) profiles used for idealized experiments. (b) The roll growth rate (normalized by $s_{0}$ ) as a function of $H$ (normalized by $h_{0}$ ). (c)-(e) As in Figs. $4 \mathrm{~d}-\mathrm{f}$, but demonstrating the change of roll structure with decreasing $H / h_{0}$; (c) $H / h_{0}=20$, (d) $H / h_{0}=3$, and (e) $H / h_{0}=1$. Dashed lines in (c)-(e) indicate the range of the vertical layer with the most significant shear $\left(z / h_{0}= \pm 1\right)$. 
where $h_{0}$ is a height scale indicating the thickness of the shear layer, $\delta u$ is a velocity scale, and $s_{0}$ is given by $\delta u / h_{0}$; $h_{0}$ and $s_{0}$ are set to $0.15 \mathrm{~km}$ and $0.03 \mathrm{~s}^{-1}$, respectively. We assume the flow is inviscid and the turbulent diffusion is set to zero in the linear SRM. The top boundary of the SRM model is at $z=3 \mathrm{~km}$, and the bottom boundary is at $z=-H$, where $H$ is the distance between the inflection point (at $z=0$ ) and the bottom boundary and is varied in the experiments $(H$ is set to $\{1,2,3,4,5,10$, $20\} \times h_{0}$ ). No-slip boundary conditions are applied at both upper and lower boundaries. Figure $6 \mathrm{~b}$ indicates the roll growth rate decreases as the bottom boundary gradually approaches the shear layer $\left(H / h_{0} \text { gets smaller }\right)^{8}$ Such a reduction is due to the change of the roll streamlines within the shear layer. Figure $6 \mathrm{c}$ shows when $H$ is sufficiently large, the streamlines of rolls on both the left and right sides of each roll are tilted against the sheared flow, resulting in prevailing negative values of $w^{\prime} u^{\prime}$ in the layer with the most significant shear (indicated by dashed lines in Fig. 6c) and thus a positive shear production term [(4)]. As $H$ gets smaller, the roll streamlines at lower levels $(z<0)$ are deformed by the bottom boundary (Figs. 6d,e). Particularly, the streamlines on the right side of each roll become less tilted. As a result, the positive values of $w^{\prime} u^{\prime}$ become more dominant within the shear layer, leading to a reduction of the shear production term $[(4)]$ and thus the roll growth rate (Fig. 6b). These results from the classic shear instability problem help explain the behavior of rolls under the HBL. The rolls in a deeper radial shear layer in the hurricane condition have a larger vertical scale, and therefore, the lower branches of their streamlines are more significantly affected by the bottom boundary, resulting in a smaller roll growth rate (Figs. $4 d-f)$.

\section{Conclusions}

We conducted the first modeling study investigating the characteristics of linear-phase rolls under a moving hurricane by embedding the linear SRM into a 3D diagnostic HBL model. It is found that the roll horizontal wavelength is determined by the radial-shear-layer depth, consistent with the finding of GG14. The roll growth rate is not only affected by the radial wind shear magnitude but also by the radial-shear-layer depth. We

\footnotetext{
${ }^{8}$ The velocity distribution given by (5) is dynamically unstable due to the presence of the inflection point at $z=0$ (Fig. 6a). The linear SRM detects and resolves the fastest-growing unstable mode. For consistency, we will refer the fastest-growing unstable mode obtained by the linear SRM as rolls.
}

find that a larger shear-layer depth has a negative impact on the roll growth rate. The bottom boundary constrains the near-surface roll streamlines and reduces the efficiency of rolls in extracting KE from the mean sheared flow. The rolls under a deeper shear layer have larger size and thus are more affected by the bottom boundary. The effect of the radial-shear-layer depth on the roll growth rate revealed in this study was not reported previously. Under a stationary and axisymmetric HBL, the shear-layer depth increases as the radial shear magnitude decreases with radius; the two factors act in concert to reduce the growth rate of rolls. This is likely why the negative impact of shear-layer depth on the roll growth rate was not noticed by previous studies (e.g., GG14).

These results improve our understanding of the main factors affecting the structure and growth of rolls in hurricanes and will provide guidance for interpreting the spatial distribution of rolls in observations and future real-storm high-resolution simulations. The real-world hurricanes are rarely axisymmetric, and this study suggests that the storm asymmetric structure, particularly of the hurricane radial wind, affects the spatial distribution of rolls. The roll-horizontal-wavelength (spatial scale) spatial distribution resembles the asymmetric pattern of the radial-shear-layer depth. The roll growth rate is affected by two factors related to the radial wind distribution: the magnitude of radial wind shear and the depth of the radial shear layer. Rolls that have larger growth rates during the linear phase are more efficient in extracting the kinetic energy from the mean shear. They can reach larger magnitudes in the nonlinear (quasi equilibrium) phase (Gao and Ginis 2016) and therefore induce larger vertical momentum fluxes and cause larger modifications to the mean wind. Future work should focus on the evolution of nonlinear-phase rolls and their interactions with the mean wind under a moving HBL. In this study, we only examined the impact of asymmetric radial flow induced by surface friction on rolls. The lower-level inflow resulting from diabatic heating is not considered in our idealized HBL model. We expect this additional inflow to further modulate the radial shear and thus affect the roll characteristics. Such an effect should be investigated with SRM embedded in a full-physics hurricane model [such as COAMPS for Tropical Cyclones (COAMPS-TC) used in Gao et al. (2017)] in future studies.

Acknowledgments. This research was funded by the Office of Navy Research through Award N000141612065. We thank three anonymous reviewers for providing comments that significantly improved this paper. 


\section{REFERENCES}

Carpenter, J. R., E. W. Tedford, E. Heifetz, and G. A. Lawrence, 2013: Instability in stratified shear flow: Review of a physical interpretation based on interacting waves. Appl. Mech. Rev., 64, 060801, https://doi.org/10.1115/1.4007909.

Ellis, R., and S. Businger, 2010: Helical circulation in the typhoon boundary layer. J. Geophys. Res., 115, D06205, https://doi.org/ 10.1029/2009JD011819.

Foster, R. C., 2005: Why rolls are prevalent in the hurricane boundary layer. J. Atmos. Sci., 62, 2647-2661, https://doi.org/ 10.1175/JAS3475.1.

Gao, K., and I. Ginis, 2014: On the generation of roll vortices due the inflection point instability of the hurricane boundary layer flow. J. Atmos. Sci., 71, 4292-4307, https://doi.org/10.1175/ JAS-D-13-0362.1.

, and - 2016: On the equilibrium-state roll vortices and their effects in the hurricane boundary layer. J. Atmos. Sci., 73, 1205-1222, https://doi.org/10.1175/JAS-D-15-0089.1.

$\longrightarrow,-$ J. D. Doyle, and Y. Jin, 2017: Effect of boundary layer roll vortices on the development of an axisymmetric tropical cyclone. J. Atmos. Sci., 74, 2737-2759, https://doi.org/10.1175/ JAS-D-16-0222.1.

Holland, G. J., 1980: An analytic model of the wind and pressure profiles in hurricanes. Mon. Wea. Rev., 108, 1212-1218, https:// doi.org/10.1175/1520-0493(1980)108<1212:AAMOTW>2.0.CO;2.

Kepert, J. D., 2001: The dynamics of boundary layer jets within the tropical cyclone core. Part I: Linear theory. J. Atmos. Sci., 58,
2469-2484, https://doi.org/10.1175/1520-0469(2001)058<2469: TDOBLJ $>2.0 . \mathrm{CO} ; 2$.

— within the tropical cyclone core. Part II: Nonlinear enhancement. J. Atmos. Sci., 58, 2485-2501, https://doi.org/10.1175/ 1520-0469(2001)058<2485:TDOBLJ > 2.0.CO;2.

Lorsolo, S., J. L. Schroeder, P. Dodge, and F. Marks, 2008: An observational study of hurricane boundary layer small-scale coherent structures. Mon. Wea. Rev., 136, 2871-2893, https:// doi.org/10.1175/2008MWR2273.1.

Morrison, I., S. Businger, F. Marks, P. Dodge, and J. Businger, 2005: An observational case for the prevalence of roll vortices in the hurricane boundary layer. J. Atmos. Sci., 62, 2662-2673, https://doi.org/10.1175/JAS3508.1.

Nakanishi, M., and H. Niino, 2012: Large-eddy simulation of roll vortices in a hurricane boundary layer. J. Atmos. Sci., 69 , 3558-3575, https://doi.org/10.1175/JAS-D-11-0237.1.

Wang, S., and Q. Jiang, 2017: Impact of vertical wind shear on roll structure in idealized hurricane boundary layers. Atmos. Chem. Phys., 17, 3507-3524, https://doi.org/10.5194/ acp-17-3507-2017.

Wurman, J., and J. Winslow, 1998: Intense sub-kilometer boundary layer rolls in Hurricane Fran. Science, 280, 555-557, https:// doi.org/10.1126/science.280.5363.555.

Zhang, J. A., K. B. Katsaros, P. G. Black, S. Lehner, J. R. French, and W. M. Drennan, 2008: Effects of roll vortices on turbulent fluxes in the hurricane boundary layer. Bound.-Layer Meteor., 128, 173-189, https://doi.org/10.1007/s10546-008-9281-2. 